\title{
Projection moiré measurement of glass specimens retrofitted with safety film
}

\author{
A. Shulev ${ }^{* a}$, W. Van Paepegem ${ }^{b}$, E. Stoykova ${ }^{a}$, S. De Pauw ${ }^{b}$, J. Degrieck $^{b}$ and V. Sainov ${ }^{\mathrm{a}}$ \\ ${ }^{\text {a }}$ Central Laboratory of Optical Storage and Processing of Information (CLOSPI-BAS), \\ Bl. 101, Akad. G. Bonchev St., Sofia 1113, Bulgaria \\ ${ }^{\mathrm{b}}$ Ghent University, Dept. of Mechanical Construction and Production, \\ Sint-Pietersnieuwstraat 41, 9000 Gent, Belgium
}

\begin{abstract}
Protection of buildings and critical public infrastructure against blast load has been recently improved by retrofitting glass windows with a safety film. As the exact physical mechanisms of the interaction between glass and safety film are not quite well understood, intensive research is conducted on the properties of this assembly. The loadings on the glass/film assembly are typically dynamic (blast, wind pressure, impact), so the lab tests are done on a drop weight setup, where a mass is falling on a retrofitted glass plate. In this work, the drop weight setup was combined with pattern projection (moiré) technique to study the time history of the out-of-plane deformations of the glass/film assembly. The fringe pattern, projected on the back side of the specimen, was generated by means of a sinusoidal phase grating under divergent high intensity infrared illumination. The whole process was recorded with a high speed camera. Local routines based on Fast Fourier Transform were used to process the captured images, and to extract the phase. The exact out-ofplane displacements were calculated by means of calibration based on previous shape measurements of several different objects with known dimensions.
\end{abstract}

Keywords: drop weight, pattern projection, Fourier analysis

\section{INTRODUCTION}

Since the terrorist attacks in 2001, a lot of important government buildings, embassies and public infrastructure (railway stations, airports) are protected against blast load. Most of these buildings have standard glazing with float glass, and replacing all float glass with tempered or laminated glass would be too expensive. Therefore the glass windows are retrofitted with a safety film, a thermoplastic optically transparent polyester film with a very large deformation capability, that is applied to the interior side of the glass window by means of a pressure sensitive adhesive.

In almost every situation, when the glass window is impacted by a hurricane, a bomb blast, burglary, a person falling in the window, etc., the security film is dynamically loaded, bridging the cracks in the glass pane and keeping all glass fragments together. Therefore, dynamic testing is also used in laboratory conditions to study the mechanical behaviour of security films. In order to validate the numerical simulations, it is important to instrument the dynamic test set-ups as widely as possible. Besides the use of dynamic loadcells and accelerometers, the registration of the instantaneous out-ofplane deflections of the glass window retrofitted with security film, provides important information for the finite element simulations.

Registration of the time history of the processes during the impact experiment on the glass/film assembly is crucial for evaluation and further optimization of protective properties of the safety films. Remote non-destructive testing by optical methods is especially suitable for solving such a complicated technical task ${ }^{1,2}$. Among existing optical methods, the techniques which rely on functional relationship of the sought object data with the phase of a periodic fringe pattern projected onto and reflected from the object occupy a special place as a full-field metrological means with non-complex set-ups and processing algorithms that are easy to implement in outdoor and industrial environment ${ }^{3-12}$. The time-scale of the observed processes implies implementation of single shot phase retrieval. As the glass/film assembly is a flat object and the normal displacements induced by the impact are small, an effective approach for phase retrieval from a

* Corresponding author (E-mail: assen@imbm.bas.bg). 
single fringe pattern is to use Fourier transform profilometry for fringe analysis ${ }^{13-20}$. It is exceptionally suitable for dynamic processes investigation ${ }^{21}$.

In this paper, the pattern projection technique has been applied to a drop weight test set-up, where a circular glass pane with security film is impacted by a falling dead weight. The fringe pattern, projected on the back side of the specimen, was generated by means of a sinusoidal phase grating. High intensity infrared divergent illumination was produced by a powerful $(1 \mathrm{~W}) \mathrm{CW}$ multimode diode laser. The whole process was recorded by a high speed camera. Local routines based on Fast Fourier Transform were used to process the captured images, and to extract the phase. The exact out-ofplane displacements were calculated by means of calibration based on previous shape measurements of several different objects with known dimensions.

\section{MATERIALS AND EXPERIMENTAL SET-UP}

\subsection{Drop weight setup}

The drop weight set-up is schematically presented in Fig. 1. A dead weight is falling down along two metal guidings. The circular glass plate (4 to $6 \mathrm{~mm}$ thick) retrofitted with safety film is mounted in a circular steel tube on the ground. When the impactor hits the glass plate this film prevents the glass fragments from shattering around. The circular steel support was designed taking into account the required parameters of the optical set-up for pattern projection. In particular, openings in the tube had been foreseen to project the fringes from one side and to observe the fringe pattern from the other side of the tube.
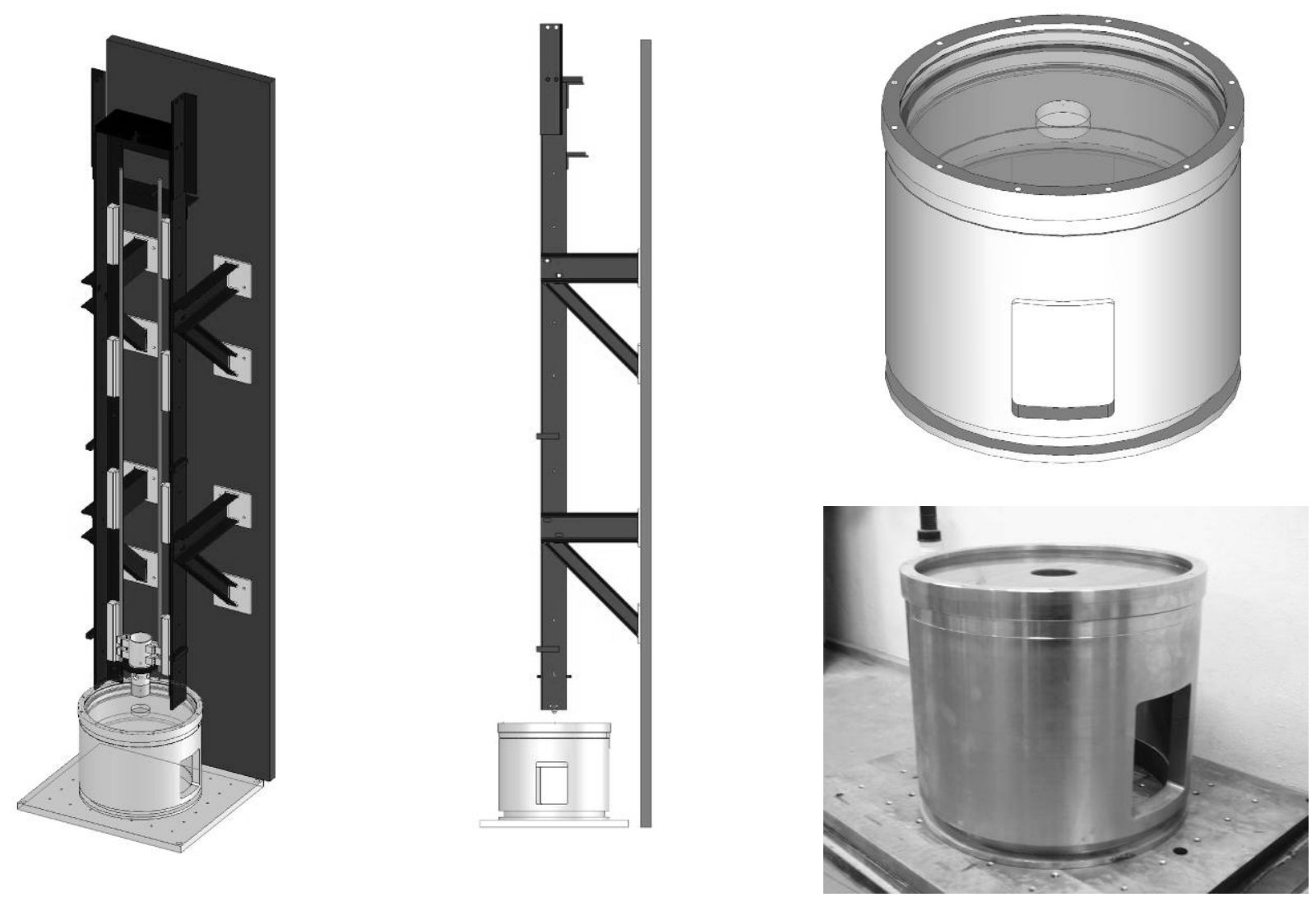

Fig. 1. Drop weight set-up for impact on circular glass specimens. 
The impactor has a weight of approximately $7 \mathrm{~kg}$ and is equipped with a hemispheric steel indentor. The drop height can range from a few centimeters up to 3 meters. The impactor is instrumented with a dynamic loadcell and an accelerometer, to measure the time history of dynamic load and acceleration/deceleration. Special precautions have been taken to protect the loadcell and the accelerometer with their cables against the glass shards. The circular glass pane has a diameter of $470 \mathrm{~mm}$ and is supported by the steel tube. A special clamping system has been designed to avoid any glass fragments escaping from the set-up. All glass fragments are confined in the area below the glass pane. There is also a braking system to decelerate the impactor when it has penetrated the glass pane. Especially for tests without security film, this braking system is very important to decelerate the impactor properly. The rectangular cut-outs of the supporting steel tube allow observing the glass pane from below through mirrors in the bottom of the supporting tube. These mirrors are protected from the falling glass shards by a plexiglass plate.

\subsection{Pattern projection technique}

Despite the alleged simplicity of pattern projection technique, adjustment of all optical elements within the drop-weight set-up turned out to be a difficult task. The constraints in space, projecting and observing angles, avoiding vibrations during the impact, etc. were extremely stringent. In particular, fixed locations and dimensions of the illumination and observation openings in the steel tube restricted the projection and observation angles. To overcome these obstacles two additional mirrors were used. Projection through one of the side openings of a fringe pattern which should cover a large area of the investigated glass plate entails divergent illumination. Finally, the complete set-up for out-of-plane measurement of a glass plate subjected to a load impact is shown in Fig.2.

It should be noted that, due to the very limited range of projection and observation angles, it was impossible to illuminate the complete back-side of the circular glass plate. Only the part, ranging from the edge to a little beyond the centre of the circle was used in the measurement. However, because of the circular symmetry of the loading, the data derived from the observed area are relevant for characterization of the impact process.

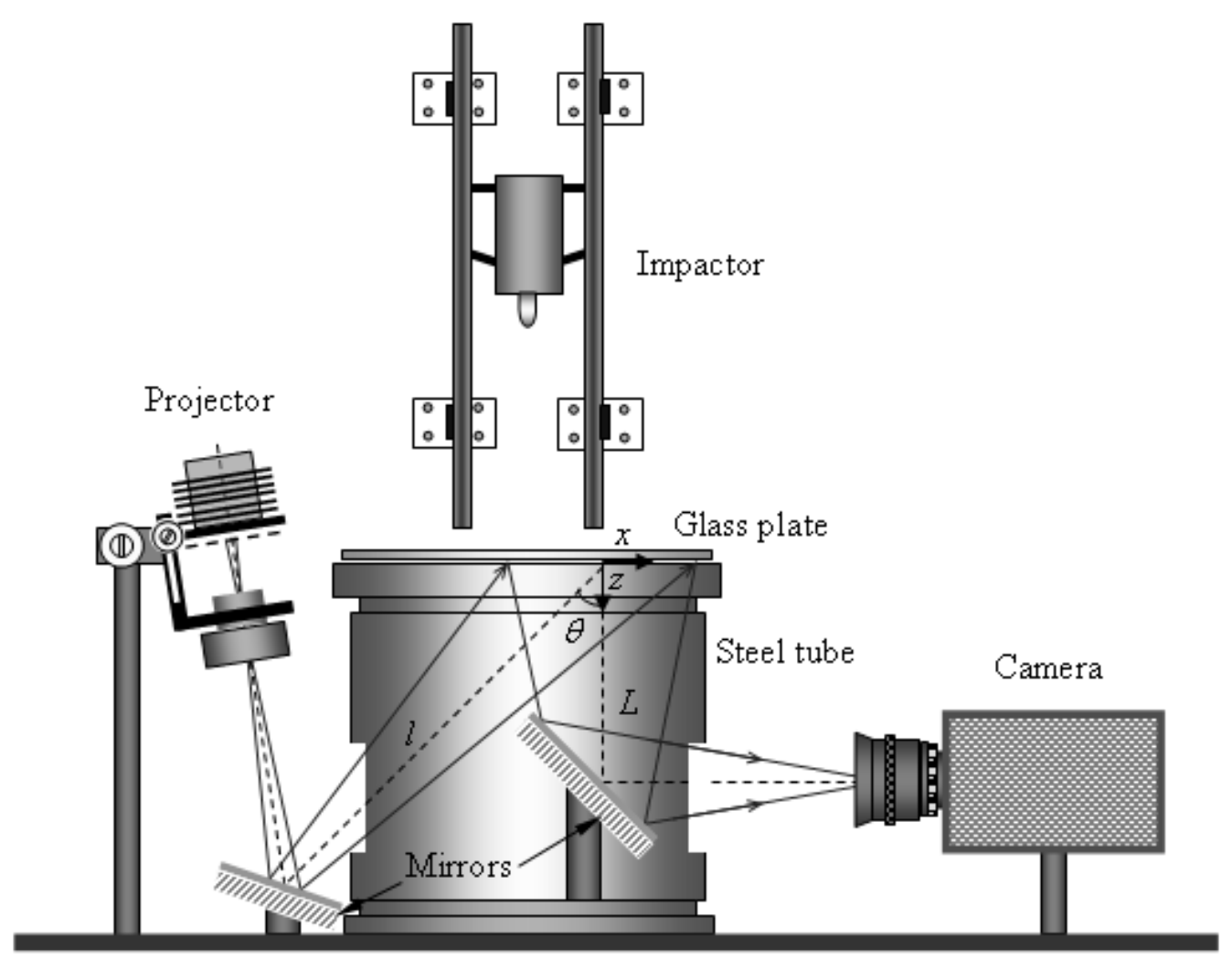

Fig.2. Optical set-up for fringe projection onto the glass plate. 
The high speed camera is a Photron Ultima APX-RS with 10-bit CMOS (Bayer system color, single sensor) with $17 \mu \mathrm{m}$ pixels. The frame rate ranges from 60 fps to 250,000 fps. Here a frame rate of 3,000 fps was chosen, as the highest frame rate with maximum resolution maintained (1024 x 1024 pixels).

A photo of the whole experimental set-up is presented in Fig. 3.

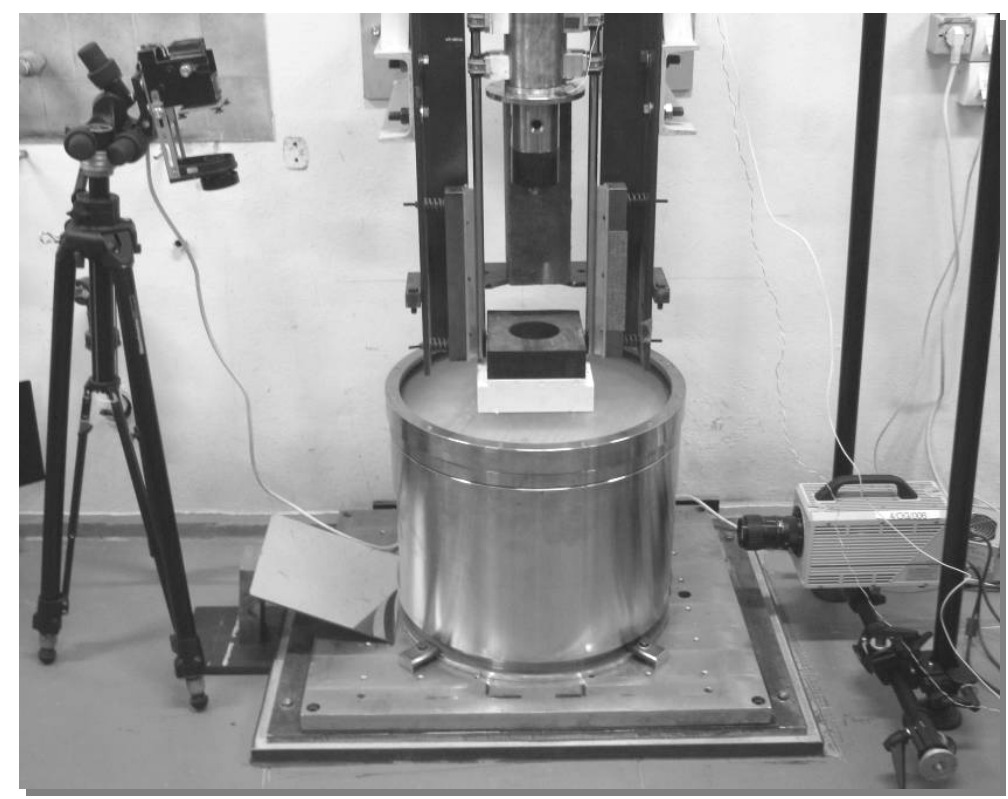

Fig.3. Photo of the whole set-up for the projection moiré measurements

The schematic presentation of the designed pattern projection device, which is denoted as "Projector" in Fig.2, is shown in Fig. 4. The light source was a CW high-power (1W) infrared diode laser $(810 \mathrm{~nm})$ which provides flicker-free light of constant intensity. Other advantages of the infrared illumination are the low thermal heating and the high CMOS sensor sensitivity in the infra red (IR) range. A sinusoidal phase grating with spacing $0.2 \mathrm{~mm}$ was used for fringe generation. The grating was produced by recording of interference patterns of two collimated beams from a Michelson interferometer onto a high resolution holographic plate HP-650, laboratory production of CLOSPI-BAS (Bulgaria). An adjustable Michelson interferometer provides equidistant sinusoidal interference fringes, whose period could vary in broad limits. The light source was a He-Ne laser $(\lambda=632.8 \mathrm{~nm}, 30 \mathrm{~mW})$. The development of the grating was realized with a fixing developer providing formation of colloidal silver grains, practically phase recording, low level of noise and high diffraction efficiency up to $70 \%$ in NIR. The required modulation of the recorded interference patterns was achieved by proper selection of exposures to fall into the dynamic range $0.5-1.5 \mathrm{~mJ} / \mathrm{cm}^{2}$.
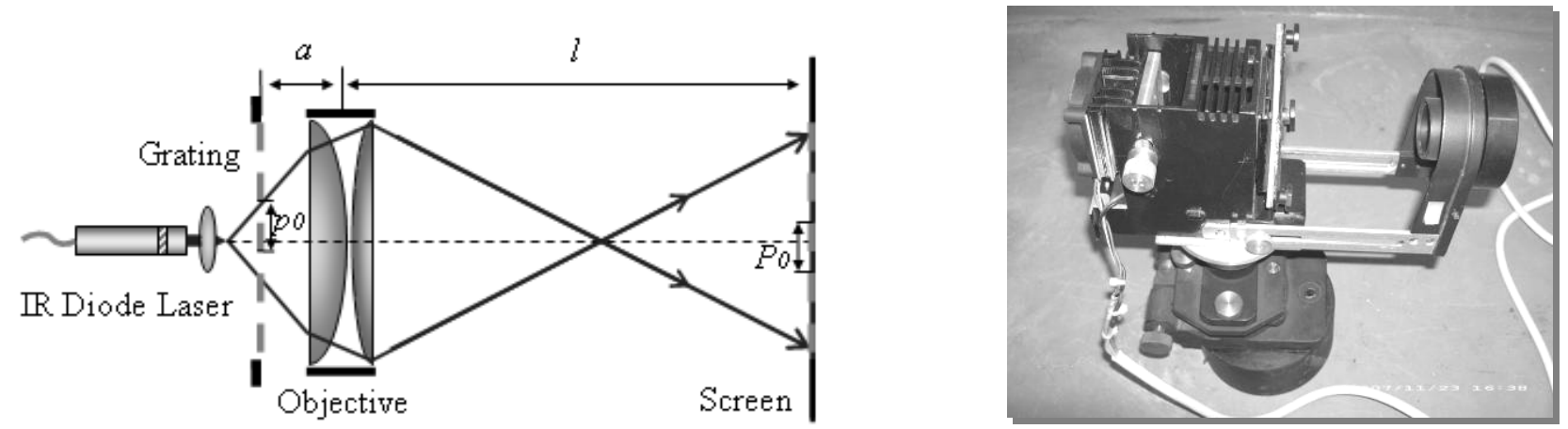

Fig.4. Fringe projection device. Schematic presentation (a), picture of the device (b). 
In general, the fringe pattern created by a thin sinusoidal phase grating is not sinusoidal. At plane-wave illumination, the diffraction pattern along transversal and longitudinal direction consists of alternating zones with a phase-reversed contrast that are separated by the so called Talbot planes in which self-imaging occurs and the contrast of fringes is zero. However, if divergent illumination is used the number of Talbot planes may be reduced to one, and fringes with practically constant contrast are observed in the longitudinal direction. The contrast of fringes increases with grating modulation but the same is true for the amplitudes of higher harmonics in the fringe pattern. Hence to evaluate the quality of the generated fringes at divergent illumination and to choose the optimal grating modulation, we calculated the intensity profile of the projected fringes using Fresnel diffraction integral ${ }^{22}$.

A thin sinusoidal phase grating located in the $(\mathrm{x}, \mathrm{y})$ plane with grating lines parallel to the $\mathrm{Y}$ axis is characterized with transmittance $^{22}$ :

$$
\tau(x, y)=\exp \left[j m \sin \left(2 \pi \frac{x}{p_{o}}\right)\right]=\sum_{q=-\infty}^{\infty} J_{q}(m) \exp \left(j 2 \pi q \frac{x}{p_{o}}\right)
$$

where $m$ is the modulation parameter, $p_{0}$ is the grating spacing along the $\mathrm{X}$ axis $\left(f_{x}=1 / p_{o}\right)$, and $J_{\mathrm{q}}$ is a Bessel function of the first kind, order $q$. For convenience, we considered the two-dimensional problem; its generalization to three dimensions is straightforward. If a grating is illuminated by a monochromatic point source at distance $s$ in front of it, the complex amplitude of the light field in the plane just behind the grating at $z=0$ in paraxial approximation of the spherical wave is given by:

$$
U\left(x_{0}, y, 0\right)=A_{0} \tau\left(x_{0}\right) \exp \left(j \frac{k}{2 s} x_{0}^{2}\right)
$$

where $A_{0}$ is a constant amplitude, $k=2 \pi / \lambda, \lambda$ is the wavelength of light and $x_{0}$ is the $x$ coordinate in this plane. After solving the Fresnel diffraction integral, we obtain for the light intensity at distance $z$ behind the grating the expression:

with

$$
I(x, z)=\frac{2 A_{0}^{2} s}{z \lambda(z+s)}\left[S_{1}^{2}(x, z)+S_{2}^{2}(x, z)\right]=\frac{2 A_{0}^{2} s}{z \lambda(z+s)} I_{S}(x, z)
$$

$$
S_{1}=\sum_{q=-\infty}^{\infty} J_{q}(m) \cos \left[\frac{2\left(\pi \sigma_{q}\right)^{2}}{k \eta}\right], S_{2}=\sum_{q=-\infty}^{\infty} J_{q}(m) \sin \left[\frac{2\left(\pi \sigma_{q}\right)^{2}}{k \eta}\right], \quad \sigma_{q}=\frac{q}{p_{o}}-\frac{x}{z \lambda} \text { and } \eta=\frac{1}{s}+\frac{1}{z} \text {. }
$$

The term in the square brackets in (3) can be written as follows:

$$
I_{S}=\sum_{q=-\infty}^{\infty} J_{q}^{2}+\sum_{\substack{q=-\infty \\ p=-\infty \\ p \neq q}}^{\infty} J_{q} J_{p} \cos \left[\left(q^{2}-p^{2}\right) \alpha-2(q-p) \beta\right]
$$

where we have introduced the notations $\alpha=\frac{\pi \lambda z s}{p_{o}{ }^{2}(z+s)}, \beta=\frac{\pi x s}{p_{o}(z+s)}$. Having in mind that $J_{-q}=(-1)^{q} J_{q}$, we obtain:

$$
\begin{gathered}
I(x, z)=\frac{2 A_{0}^{2} s}{z \lambda(z+s)}\left\{I_{0}(z)+I_{V}(z) \sin \frac{2 \pi x s}{p_{o}(z+s)}+\Theta(x, z)\right\} \\
I_{0}(z)=\sum_{q=-\infty}^{\infty} J_{q}^{2} \quad I_{V}(z)=2\left\{\sum_{q=0}^{\infty} J_{2 q} J_{2 q+1} \sin (2 q+1) \alpha+\sum_{q=1}^{\infty} J_{2 q} J_{2 q-1} \sin (2 q-1) \alpha\right\} \\
\Theta(x, z)=2 \sum_{q=0}^{\infty} \sum_{p=1}^{\infty}\left[J_{2 q} J_{2 q+2 p+1} \sin (4 p \beta) \times \sin \{\alpha(2 p+1)(2 p+4 q+1)\}+\cos (4 p \beta) \times\left(J_{2 q} J_{2 q+2 p} \cos \{4 \alpha(q+2 p)\}+J_{2 q} J_{2 q+2 p+1} \cos \{4 \alpha(q+2 p+1)\}\right)\right.
\end{gathered}
$$

We see that the intensity distribution consists of sinusoidal terms which are responsible for the periodicity along $\mathrm{Z}$ axis and $\mathrm{X}$ axis: 


$$
\frac{1}{s}+\frac{1}{\Lambda_{z}^{q p}}=\left|q^{2}-p^{2}\right| \frac{\lambda}{2 p_{o}^{2}} \quad \Lambda_{x}^{q p}=\frac{L}{|q-p|}\left(1+\frac{z}{s}\right)
$$

where $\Lambda_{z}^{q p}$ and $\Lambda_{x}^{q p}$ are the spatial periods in z-direction and $x$-direction for the given $q$ and $p$.

Fig.5 depicts variation of the fringe contrast with the distance from the grating for different locations of the point source at $m=0.2$. At this value of the modulation parameter the first harmonic dominates everywhere but the height of its peak along $z$ is a sum of a constant background and an oscillating term. Talbot planes of grating self-imaging are clearly seen when the source is far from the grating. It is clear that by a proper choice of the distance $s$ it is possible to create fringes of the same contrast in a large region along the axis of beam propagation, $\mathrm{Z}$.

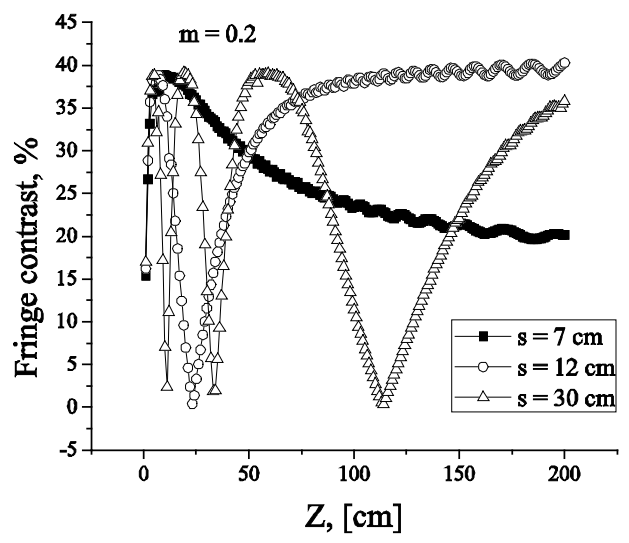

Fig. 5. Fringe contrast as a function of the distance from the grating, $\lambda=810 \mathrm{~nm}, p_{0}=0.2 \mathrm{~mm}$.

The theory that presents a diverging moiré fringe projection is well known and has been described in the literature ${ }^{12}$. The intensity distribution along the surface of the investigated object can be represented by the following equation:

$$
I(x, y)=A(x, y)+B(x, y) \cos (2 \pi \phi(x, y)+2 \pi \psi(x, y)),
$$

where $A(x, y)$ is a slowly varying function representing the background illumination, $B(x, y)$ is a slowly varying function representing the contrast between light and dark fringes, $\phi(x, y)$ is the phase of the grating on the surface of the object and $\psi(x, y)$ is the phase of the object. From the geometry of the optical setup shown in Fig. 2 where the optical axis of the projector and camera cross the undeformed surface at a point which defines the centre of the coordinate system the phase of the grating and the object can be described as:

$$
\phi(x, y)=\frac{x}{P_{0}}\left(1+x \frac{\sin \theta}{l}\right)^{-1}, \quad \psi(x, y)=\frac{z(x, y)}{P_{0} \cos \theta}\left(\sin \theta+x \frac{(L-l \cos \theta)}{L l}\right)\left(1+x \frac{\sin \theta}{l}\right)^{-2},
$$

where $z(x, y)$ is the surface height of the object, $\theta$ is the angle between the optical axes of the projection and camera lenses, $l$ is the distance from the projection lens to the origin of the coordinate system, $L$ is the distance from the camera lens to the origin of the coordinate system, and $P_{0}$ is the period of the projected grating at $x=0$. The previous equations are valid if the grating period $p_{0}$ is much smaller than the distance $a$ between the lens and the projection grating $\left(p_{0}\right.$ « $\left.a\right)$ and if the maximal surface height of the object $z_{\max }$ divided by the cosine of the angle $\theta$ is much smaller than the distance $l\left(z_{\max } / \cos \theta \ll l\right)$. 


\section{NUMERICAL RECONSTRUCTION}

Phase distribution of the projected grating on the surface of the investigated object is not a linear function of $x$ which can be seen from Equation (8). It means that the period of this fringe pattern varies along the object. This nonlinearity complicates additionally the phase retrieval if the Fourier transform approach is applied directly. Therefore a different method which treats the fringe pattern in a local manner assuming linearity in a given small region of the fringe pattern is more appropriate. Hence to extract the phase from the obtained moiré fringe patterns, numerical algorithms based on Local Fourier Transformations ${ }^{23}$ or on the so called Windowed Fourier Transform ${ }^{24}$ were used. The principle of this method relies on a movable window function which multiplies the investigated fringe pattern forming in this way many local subfringe patterns. Next the Fourier spectra of each local subfringe pattern are calculated. This can be presented by:

$$
\mathrm{I}_{A B}(u, v, a, b)=\mathfrak{J}\left\{I(x, y) W_{A B}(x-a, y-b)\right\},
$$

where $\mathfrak{I}$ is the Fourier transform, $u$ and $v$ are the spatial frequencies, $I(x, y)$ is the intensity distribution recorded by the camera and it can be represented theoretically by Equation (7), $W_{A B}(x-a, y-b)$ is the window function, $A$ and $B$ correspond to the window dimensions, $a$ and $b$ are the shifting factors and $\mathcal{J}_{A B}(u, v, a, b)$ is the local spectrum selected from this window function. The window function can be Gaussian, Hanning, Hamming, rectangular, and so on. In this paper we consider a rectangular window function:

$$
W_{A B}(x-a, y-b)=\operatorname{rect}\left(\frac{x-a}{2 A}, \frac{y-b}{2 B}\right),
$$

where the dimensions of the window are $2 A \times 2 B$.

For each local Fourier spectrum half band frequency filtering ${ }^{15}$, zero term and weak frequency suppression and inverse Fourier transformation are applied:

$$
I_{A B}(x, y, a, b)=\mathfrak{I}^{-1}\left\{T_{t h r}\left\{I_{A B}(u, v, a, b) \operatorname{rect}\left(\frac{x-a}{A}-1, \frac{y-b}{2 B}\right)\right\}\right\},
$$

where $T_{t h r}$ is the thresholding operator which can be presented as:

$$
T_{\text {thr }}\{x\}=\begin{aligned}
& x \text { if } x \geq t h r \\
& 0 \text { if } x<t h r
\end{aligned}
$$

where thr is the threshold which can be estimated from the noise level in the fringe pattern individually for each local subfringe pattern ${ }^{25,26}$. This technique perfectly reduces the noise separately in each part of the fringe pattern. The phase distribution can be represented by:

$$
\psi(x, y)=\arctan \frac{\operatorname{Im}\left(\iint I_{A B}(x, y, a, b) d a d b\right)}{\operatorname{Re}\left(\iint I_{A B}(x, y, a, b) d a d b\right)}
$$

The optimal window size depends on the specificity of the fringe pattern, mainly on the fringe density and noise level. The presented phase extracting procedure is reliable for sophisticated fringe patterns and extremely robust against strong presence of noise which is typical for the high speed cameras. By means of the proposed algorithm phase maps extracted from the captured fringe patterns during the impact are subtracted from the initial phase map corresponding to the object's surface before the impact. The obtained phase maps can be unwrapped by means of different unwrapping algorithms ${ }^{27}$. At the end of the impact the glass plate is broken and therefore there are zones with lack of phase information. We have chosen $\mathrm{Z} \pi \mathrm{M}$ algorithm ${ }^{28}$ because it works well in the presence of singularity zones and can interpolate them naturally. 


\section{RESULTS}

The accuracy of the proposed measurement technique has been verified first with a dome with known height and diameter. Captured fringe patterns of the flat surface and surface with a dome with diameter $44 \mathrm{~mm}$ and height $4.8 \mathrm{~mm}$ are shown in Fig. 6.(a, b). The corresponding phase maps are presented in Fig.6.(c, d).

(a)

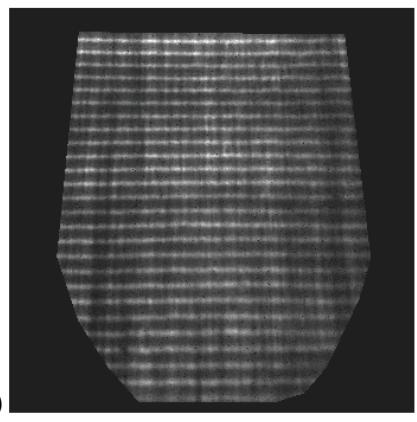

(c)

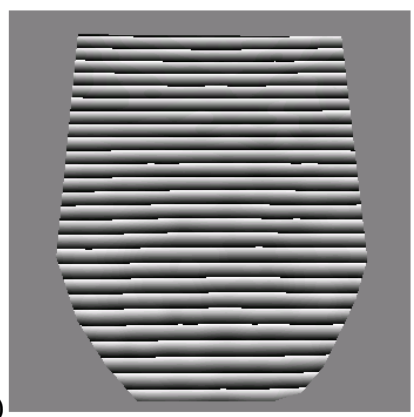

(b)

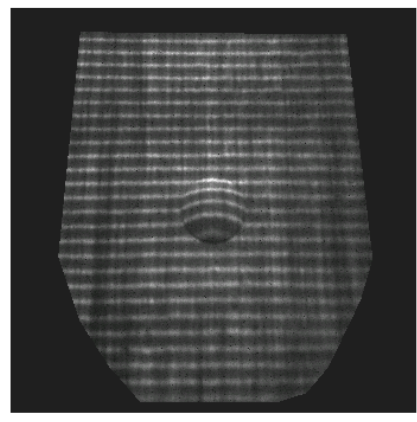

(d)

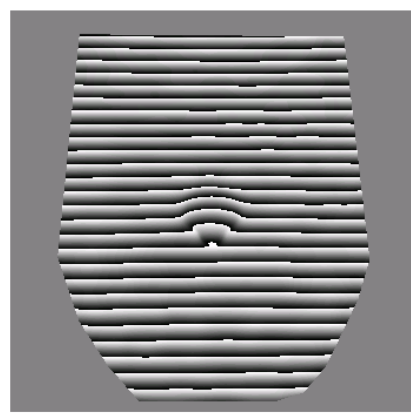

Fig.6. Fringe pattern of the reference surface (a), fringe pattern of a dome put on the reference surface. Calculated phase distribution of the reference surface (c) and the phase of the dome (d).

By means of Equation (8) from the difference between the final and initial phase distributions the dome profile has been calculated and Fig. 7.(a) shows the result. The numerically generated profile of a dome with the same shape is shown in Fig. 7. (b). Both profiles has been compared and a section across the center line is presented in Fig. 7. (c). The maximal error is observed in the regions of shadowing where the grating can not be projected and is less than 0.6 mm. Other error sources are the poor illumination and camera nonlinearity leading to a non-sinusoidal distribution of the fringe pattern. The root mean square error is approximately $0.02 \mathrm{~mm}$.

(a)

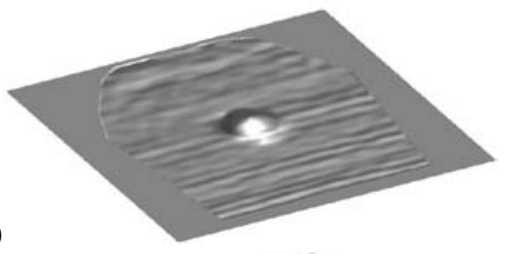

(b)

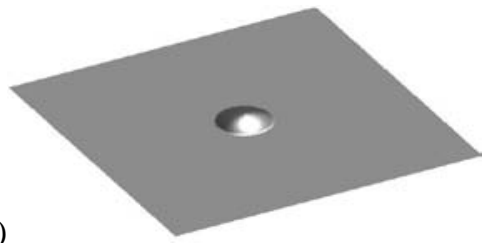

(c)

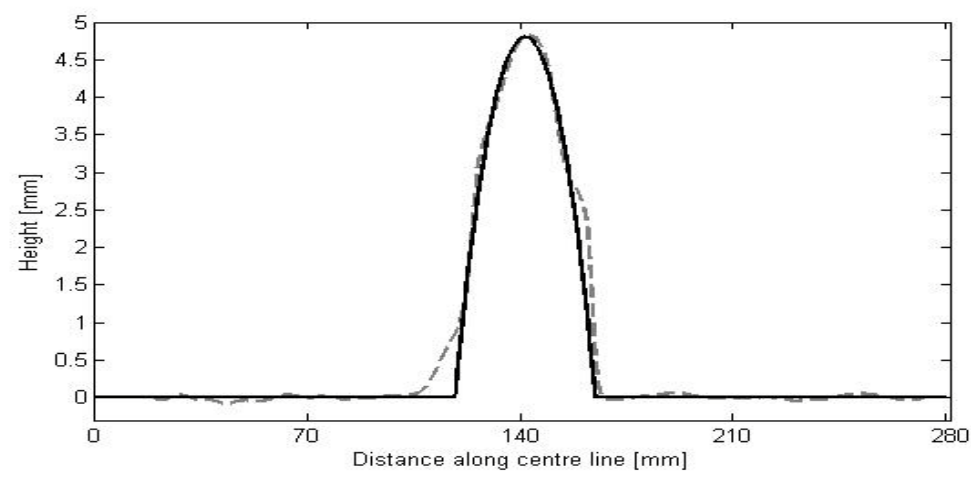

Fig.7. Calculated dome profile (a), theoretical profile (b), comparison of both surfaces along centre line of the dome (c). 
Finally the glass deformations have been studied and some of the results are shown in Fig. 8. The glass behaviour without security film subjected to a series of load impacts with dead weight falling from different heights was observed. The specimen remained unbroken until a drop height of $70 \mathrm{~cm}$. Then the glass plate was broken in many fragments and further processing of the deformation was impossible. For that reason the glass deformations are presented before the glass shattering.

(a)

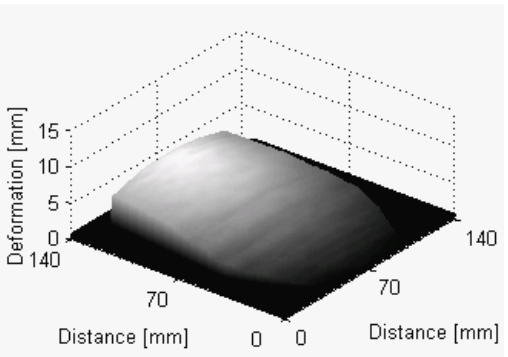

(d)

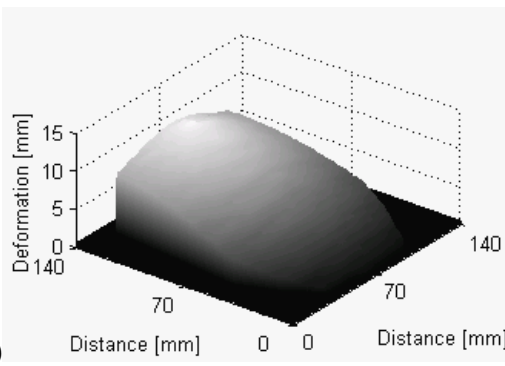

(b)

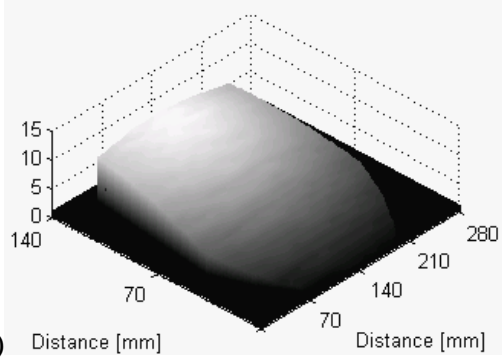

(e)

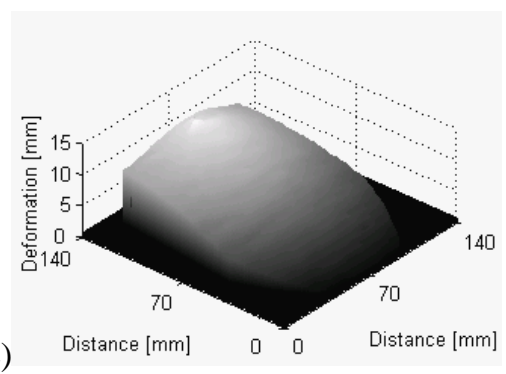

(c)

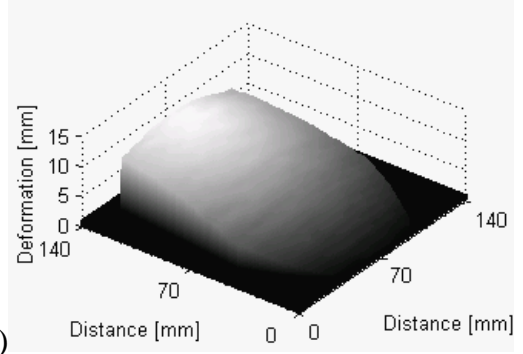

(f)

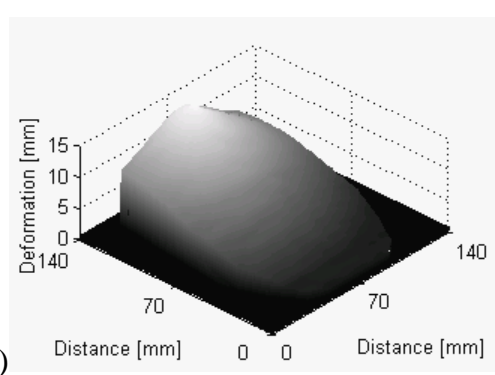

Fig. 8. Deformations of a glass plate without security film. The drop height of the impactor varies: $20 \mathrm{~cm}(\mathrm{a}), 40 \mathrm{~cm}$ (b), $60 \mathrm{~cm}$ (c), $70 \mathrm{~cm}$ while the glass is still unbroken, (d), $70 \mathrm{~cm}$ when the glass starts breaking (e), $70 \mathrm{~cm}$ when the impactor penetrates the glass

Deformation fields of a glass specimen with security film at different stages of the impact are presented in Fig. 9. Although the glass was broken when the dead weight was fallen from only $20 \mathrm{~cm}$, the glass pieces remain together. Even more the security film restores the initial flat shape of the specimen gathering all glass pieces together. This effect can be clearly seen in Fig. 9. (b, c).

(a)

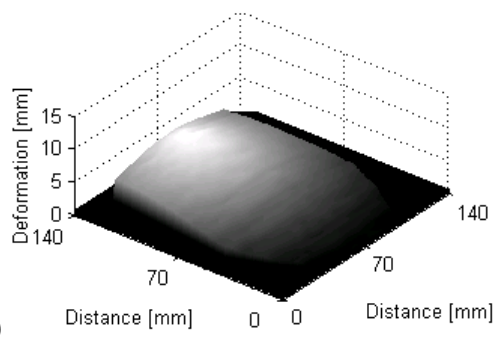

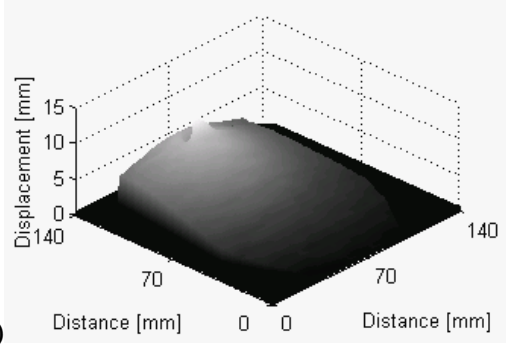

(b)

Fig. 9. Deformations of a glass plate with security film. Before the penetration of the impactor (a), after the penetration of the impactor

\section{CONCLUSIONS}

The feasibility of the pattern projection measurement has been investigated for real-time measurements of the out-ofplane displacement profile of glass plates subject to a load impact. The phase information was extracted from the captured fringe patterns by means of specialized software based on Local Fourier processing. The phase evaluation is possible due to the carrier frequency nature of the moiré patterns. Usually the high speed cameras offer low resolution 
and high noise levels which complicate most of the phase extracting algorithms. The natural filtering facilities of the proposed algorithm permit correct processing of the captured noisy data, reliability and exactness of the results. Further investigations are ongoing to increase the accuracy of the technique and to improve the automated processing of the successive digital frames.

\section{ACKNOWLEDGEMENTS}

The authors are grateful to the Fund for Scientific Research - Flanders (F.W.O.) for the bilateral collaboration between UGent (Belgium) and CLOSPI-BAS (Bulgaria).

\section{REFERENCES}

[1]. Chen F., Brown G.M., and Song M., “Overview of Three-Dimensional Shape Measurement Using Optical Methods”, Opt. Eng. 39(1), 10 - 22 (2000).

[2]. B. Dorrio and J. Fernandez, Phase-evaluation methods in whole-field optical measurement techniques, Meas. Sci. Technol. 10, R33-R55 (1999).

[3]. Post D, Han B, Ifju P., [High sensitivity moire: experimental analysis for mechanics, materials], Springer, NewYork, (1994).

[4]. Creath K. and Wyant J. C., [Moiré and fringe projection techniques], Optical Shop Testing, D. Malacara, ed. Wiley, (1992)

[5]. Rastogi P. K. (Ed.), “Holographic Interferometry Principles and Methods”, Springer Series in Optical Sciences 68, SpringerVerlag, Berlin (1994)

[6]. Kreis T., [Handbook of Holographic Interferometry], Wiley-VCH GmbH, Weinheim (2005).

[7]. Idesawa M., Yatagai T., and Soma T., "Scanning moiré method and automatic measurement of 3-D shapes,” Appl. Opt. 16, 2152 - 2162 (1977).

[8]. Yi-Bae C., and Seung-Woo K., "Phase-shifting grating projection moiré topography”, Opt. Eng., 37 (3), $1005-1010$ (1998)

[9]. Peng X., Gao Z., and Zhou S. M., "Surface contouring by a new type of digital moiré technique”. Optik, 100 (2), 63-67, (1995)

[10].Peng X., Zhu S., Su C., Tseng M., “Model-based digital moiré topography, Optik, 110 (4), 184-190, (1999)

[11].Purcell D., Davies A., and Farahi F., "Effective wavelength calibration for moiré fringe projection”, Appl. Opt, 45.(34), (2006)

[12].Gasvik K. J., [Optical Metrology], Wiley, (2002)

[13].Takeda M., Ina H, Kobayashi S. "Fourier-transform method of fringe-pattern analysis for computer based topography and interferometry”, J Opt Soc Am., 72(1), 156-60 (1982)

[14].Takeda M., Motoh K., "Fourier transform profilometry for the automatic measurement of 3-D object shapes”, Appl. Opt., 22 (24), 3977-3982 (1983).

[15].Kreis T., “Digital holographic interference-phase measurement using the Fourier-transform method”, J Opt. Soc. Am., 3 (6), $847-855$ (1986)

[16].Su X., Chen W., “Fourier transform profilometry: a review”, Opt. Las. Eng. 35, 263-284 (2001)

[17].Su X., Chen W., Zhang Q., Chao Y., “Dynamic 3-D shape measurement method based on FTP”, Opt. Las. Eng. 36, 49-64 (2001).

[18]. Li J., Su X., Guo L., “Improved Fourier transform profilometry for the automatic measurement of three-dimensional object shapes”, Opt Eng; 29 (12), 1439-44 (1990)

[19].Yi J., Huang S., "Modified Fourier transform profilometry for the measurement of 3D steep shapes”, Opt. Las. Eng, 27 (5), 493-505 (1997)

[20].Mao X., Chen W., Su X., “Improved Fourier-transform Profilometry”, Appl. Opt., 46 (5), 664 - 668 (2007)

[21].Van Paepegem W., Shulev A., Moentjens A., Harizanova J., Degrieck J., Sainov V., "Use of projection moire' for measuring the instantaneous out-of-plane deflections of composite plates subject to bird strike”, Opt. Las. Eng. 46, 527- 534 (2008)

[22].Goodman J. W., [Introduction to Fourier optics], Roberts and Company Publishers, (2004)

[23].Shulev A., Roussev I., and Sainov V., "New automatic FFT filtration method for phase maps and its application in speckle interferometry”, Proc. SPIE 4933, 323-327 (2003).

[24].Kemao Q., “Windowed Fourier transform for fringe pattern analysis”, Appl. Optics 43 (13), 2695-2702 (2004).

[25].Donoho D., and Johnstone I., “Ideal Spatial Adaptation via Wavelet Shrinkage”, Biometrica 81, 425-455 (1994).

[26].Shulev A., Gotchev A., Foi A., and Roussev I., "Threshold selection in transform-domain denoising of speckle pattern fringes”, Proc. SPIE 6252, 625220 (2006)

[27]. Ghiglia D., Pritt M., [Two-dimensional phase unwrapping: theory, algorithms and software], Wiley, New York (1998).

[28].Dias J. and Leitao J., “The Z $\pi$ M Algorithm for Interferometric Image Reconstruction in SAR/SAS”, IEEE 20 (5), (2001) 